\title{
Governança de TIC no Contexto de Cidades Inteligentes: Um Estudo Exploratório nos Municípios do Estado do RJ
}

\author{
Luiz Claudio Diogo Reis ${ }^{1}$, Flavia Cristina Bernardini², Claudia Cappelli ${ }^{3}$, \\ Simone Bacellar Leal Ferreira ${ }^{1}$ \\ ${ }^{1}$ Instituto de Informática - Universidade Federal do Estado do Rio de Janeiro \\ (UNIRIO) Av. Pasteur, 458 - Urca CEP 22290240 - Rio de Janeiro - RJ - Brasil \\ ${ }^{2}$ Departamento de Computação - Universidade Federal Fluminense (UFF) \\ - Niterói - Rio de Janeiro - RJ - Brasil \\ ${ }^{3}$ Instituto de Informática - Universidade Federal do Rio de Janeiro (UFRJ) \\ \{luiz.reis, simone\} @uniriotec.br, flavia.bernardini@ic.uff.br, \\ claudia.cappelli@gmail.com
}

\begin{abstract}
ICTs represent essential resources for supporting municipal management in providing services to citizens, especially in the context of smart cities. In this scenario, ICT governance is fundamental to guarantee a return on investments and effectiveness in the city administration. In an exploratory research in ten municipalities in the state of RJ based on governance practices adoption, it was identified precariousness in the implementation of COBIT 2019 requirements. For future actions, the municipalities must establish a strategy for managing technological resources, especially to transform cities into smart ones.

Resumo. As TICs representam recursos essenciais de apoio à gestão dos municípios na prestação de serviços aos cidadãos, especialmente no contexto de cidades inteligentes. Nesse cenário, a governança das TICs é fundamental para garantir retorno sobre os investimentos $e$ efetividade na administração das cidades. Em uma pesquisa exploratória em dez municípios do Estado do RJ sobre a adoção de boas práticas de governança foi identificado precariedade na implementação de requisitos baseados no COBIT 2019. Como ações futuras, os municípios devem estabelecer uma estratégia de gestão dos recursos tecnológicos para a transformação inteligente das cidades.
\end{abstract}

\section{Introdução}

O conceito de Cidades Inteligentes tem despertado o interesse de governantes, da iniciativa privada, de institutos de pesquisa e das pessoas em geral. Segundo [GilGárcia et al. 2015], um princípio fundamental aplicado a esse contexto refere-se ao uso da tecnologia para melhorar os serviços aos cidadãos e promover a economia sustentável, a cocriação e a participação ativa da sociedade no dia a dia das cidades. Segundo os autores, o uso de recursos de Tecnologias da Informação 
e Comunicação (TICs) podem proporcionar uma gama de soluções envolvendo, por exemplo, aplicações de Internet das Coisas, Big Data e Inteligência Artificial.

As TICs permeiam diversas áreas, domínios e setores de um município, portanto sua governança precisa ser tratada de forma holística, multidisciplinar e transversal, abrangendo todos os serviços prestados aos cidadãos. Assim, é fundamental que o planejamento estratégico de uma cidade constante do Plano Diretor esteja alinhado à governança de TIC e que os gestores e profissionais da área de TI entendam a sua importância, potencialidades e benefícios [Brasil 2001].

No âmbito da administração pública municipal, a governança de TIC tem um papel fundamental, especialmente para a transformação inteligente das cidades porque envolve investimentos expressivos e a necessidade de alinhamento entre os objetivos estratégicos da cidade e as secretarias subordinadas. No entanto, apesar da relevância da governança de TIC para as cidades inteligentes, há uma quantidade limitada de pesquisas que examinem os mecanismos de governança de TIC nesse contexto [Connolly et al. 2017]. Há poucas pesquisas com ênfase em governança e uma lacuna sobre como os governos gerenciam cidades inteligentes [Bolívar 2015]. Ainda, não houve um esforço sistemático para entender as iniciativas de governança e como ela é planejada, implementada e monitorada para obter melhor desempenho e capacidade de resposta das cidades [Pereira et al. 2018]. [Reis et al. 2020] também revelaram que o framework COBIT 5, referência para a governança de TIC nas organizações públicas e privadas, também foi precariamente aplicado no contexto de cidades inteligentes. [Boscarioli et al. 2017] descrevem que a gestão das cidades inteligentes representa um dos grandes desafios da área de Sistemas de Informação para o Brasil.

Nesse contexto, esse trabalho tem como objetivo identificar se boas práticas de governança de TIC são utilizadas pelas prefeituras das cidades do estado do Rio de Janeiro no contexto de cidades inteligentes. Essa pesquisa, de caráter exploratório, baseou-se em consultas a websites e solicitações pela Lei de Acesso a Informações [Brasil 2011] nos dez municípios melhores posicionados no ranking do Índice de Governança Municipal (IGM) mantido pelo Conselho Federal de Administração (CFA), denominado IGM-CFA [CFA 2018]. O resultado do estudo revelou que existem prefeituras que adotam práticas de governança de TIC e que determinadas práticas são adotadas em mais de uma prefeitura e que há prefeituras que não adotam quaisquer práticas.

Este trabalho está organizado da seguinte forma: Na Seção 2 é apresentada a revisão da literatura sobre cidades inteligentes, governança de TIC e boas práticas de governança; $\mathrm{Na}$ Seção 3 é apresentada a metodologia da pesquisa utilizada para a condução do estudo exploratório; Na Seção 4 são apresentados os resultados e uma análise do cenário atual nas prefeituras; $\mathrm{Na}$ Seção 5 são delineadas as conclusões do trabalho e propostas de estudos futuros.

\section{Revisão da Literatura}

\subsection{Cidades Inteligentes}

O tema das cidades inteligentes tem sido objeto de discussões da comunidade científica há algum tempo, mas pode ser considerado como abstrato e sem 
precisão conceitual [Hollands 2008] [Kobayashi et al. 2017]. Alguns autores definiram domínios para caracterizarem as cidades como inteligentes, propondo, inclusive, a sua redefinição para uma abordagem mais ampla, como cidades inteligentes e sustentáveis que utilizam recursos de TICs [Ahvenniemi et al. 2017]. Outros abordam as diferenças entre cidades inteligentes e cidades digitais, pressupondo que um sistema inteligente esteja associado a um sistema digital [Jucevicius et al. 2014]. Segundo [Caragliu et al. 2011], um sistema inteligente deve ser dotado de infraestrutura, capital humano, informação e capacidade para gerar conhecimento.

Alguns autores associam o conceito de cidades inteligentes a modelos ou frameworks [Nam and Pardo 2011]. [Gil-Gárcia et al. 2015] descrevem as principais características de cidades inteligentes em um framework composto por quatro dimensões e dez domínios: Dados e Tecnologia (Dados e Informação; TICs e outras Tecnologias); Ambiente Físico (Ambiente Construído e Infraestrutura da Cidade; Ambiente Natural e Sustentabilidade Ecológica); Sociedade (Governança, Engajamento e Colaboração; Capital Humano e Criatividade; Economia do Conhecimento e Ambiente Pró-Negócios); Governo (Serviços Públicos; Administração e Gerenciamento da Cidade; Arranjos Institucionais).

\subsection{Governança da Tecnologia de Informação e Comunicação (TIC)}

A governança de TIC está associada ao tratamento das decisões de tecnologia e como elas são orquestradas para atender às diferentes partes interessadas em uma organização [Huang et al. 2010]. Os mecanismos de governança de TIC devem ser aprovados e apoiados pela alta administração e cobrir toda a organização, de forma a sustentar suas estratégias e objetivos [Bianchi et al. 2017] [ISACA 2018]. Nesse sentido, a composição da governança de TIC pode ser baseada em estruturas (funções e responsabilidades) e em processos (melhores práticas de TIC), como COBIT e ITIL [ISACA 2018b]. [De Haes and Grembergen 2009] destacam a importância de mecanismos voltados para a participação ativa e a colaboração entre as partes interessadas. Juntos, esses elementos devem fornecer o alinhamento das atividades com práticas de TIC e prover benefícios para a organização [Héroux and Fortin 2017]. [Weill and Ross 2004] revelam que uma governança de TIC eficaz incentiva e alavanca a participação de todo o pessoal da organização, garantindo conformidade com a visão e objetivos estratégicos, de forma a alcançar um paradoxo de gerenciamento contemplando simultaneamente capacitação e controle. Esses autores evidenciaram que as organizações que adotaram práticas de governança de TIC possuem melhores retornos sobre os ativos.

Em uma outra visão, complementar à anterior, a governança de TIC abrange a descrição de políticas, estruturas e gerenciamento de processos relacionados às funções de TI, o que implica (re-)estruturação de processos, práticas de trabalho e ambiente cultural [Weill and Broadbent, 2000]. Nesse contexto, a governança de TIC pode ser caracterizada sob uma perspectiva técnica e social. A tomada de decisão e a responsabilidade de TIC são elementos centrais da governança e devem contemplar a definição de prioridades e alocação de serviços de TI, a garantia de conformidade regulatória e o gerenciamento de parceiros [ISACA 2012] [ISACA 2018b]. Segundo o [SISP 2017], a governança 
de TIC é um sistema em que o uso atual e futuro das TICs é dirigido e controlado, mediante avaliação e direcionamento de sua aplicação para dar suporte e monitorar a execução dos planos estratégicos e operacionais. A governança corporativa de TIC é exercida pelo conselho que supervisiona a implementação de processos, estruturas e mecanismos relacionais, baseados em responsabilidades definidas, alinhamento estratégico e geração de valor [ISACA 2018].

\subsubsection{Boas Práticas de Governança de TIC}

Várias diretrizes sobre como a governança de TIC pode ser implementada surgiram do meio acadêmico [De Haes and Van Grembergen 2009]. A literatura mostra um papel significativo das estruturas e padrões de governança de TIC baseados em boas práticas [De Haes et al. 2013]. Por exemplo, o framework COBIT (Objetivos de Controle em Tecnologia da Informação), desenvolvido pela ISACA (versões COBIT 5 e COBIT 2019), pode ser utilizado para a estruturação da governança corporativa [ISACA 2012] [De Haes et al. 2019] [Reis et al. 2020]. [Joshi et al. 2018] reconhecem que a implementação bem-sucedida da governança das TIC é complexa e pode ser apoiada por boas práticas para auxiliar as organizações nessa tarefa. Nesse trabalho o COBIT 2019 é apresentado como referência para a governança de TIC.

\subsubsection{COBIT 2019}

O COBIT 2019 é um framework para a governança corporativa e o gerenciamento de TIC que pode ser aplicado a organizações públicas e privadas. O modelo COBIT, desde as versões anteriores, vem sendo adotado nas organizações públicas brasileiras, especialmente por recomendação dos órgãos de controle externo [SISP 2017]. O COBIT 2019 sugere uma estrutura de processos que deve ser customizada de acordo com o porte da organização [ISACA, 2018b].

O COBIT 2019 descreve sete componentes para o sistema de governança [ISACA, 2018]. No COBIT 5 esses componentes eram denominados habilitadores [ISACA 2012] [De Haes et al. 2019]. [Reis et al. 2020] descreveram os habilitadores no contexto de cidades inteligentes e, nesse trabalho, a terminologia foi atualizada para sistema de governança [ISACA 2018], conforme Tabela 1.

Tabela 1. Descrição dos componentes do sistema de governança

\begin{tabular}{|c|l|}
\hline $\begin{array}{c}\text { Componente do Sistema } \\
\text { de Governança }\end{array}$ & \multicolumn{1}{|c|}{ Descrição } \\
\hline $\begin{array}{c}\text { Princípios, Políticas e } \\
\text { Modelos }\end{array}$ & $\begin{array}{l}\text { As cidades inteligentes devem ser concebidas a partir de diretrizes, } \\
\text { planejamento estratégico, politicas, regras, princípios e procedimentos } \\
\text { relacionados às TICs que serão utilizados como instrumentos balizadores e } \\
\text { direcionadores para atingimento das metas das cidades. }\end{array}$ \\
\hline Processos & $\begin{array}{l}\text { As cidades inteligentes devem estabelecer um modelo de referência baseado } \\
\text { em processos de TIC para atendimento aos seus objetivos estratégicos, com } \\
\text { propósitos, padrões e metas especificas. }\end{array}$ \\
\hline Estrutura Organizacional & $\begin{array}{l}\text { A estrutura administrativa das cidades inteligentes deve contemplar níveis } \\
\text { apropriados de responsabilidade, autoridade e atribuições para tomada de } \\
\text { decisão adequada sobre soluções, recursos e investimentos relacionados às } \\
\text { TICs. }\end{array}$ \\
\hline Cultura, Ética e & $\begin{array}{l}\text { As cidades inteligentes abrangem a cultura, a ética e o comportamento dos } \\
\text { cidadãos e dos profissionais de TIC, requisitos intangíveis que devem ser } \\
\text { considerados no planejamento da estratégia e desenvolvimento de soluções e } \\
\text { serviços de TIC. }\end{array}$ \\
\hline Comportamentos & Os tomadores de decisão nas cidades inteligentes necessitam de dados, \\
\hline Informação &
\end{tabular}




\begin{tabular}{|c|l|}
\hline & $\begin{array}{l}\text { informações e conhecimento compartilhados entre todas as partes } \\
\text { interessadas, de forma transparente e segura, respeitando aspectos de } \\
\text { confidencialidade e privacidade dos dados. }\end{array}$ \\
\hline $\begin{array}{c}\text { Pessoas, Habilidades e } \\
\text { Competências }\end{array}$ & $\begin{array}{l}\text { As cidades inteligentes exigem diferentes papéis e níveis de habilidades e } \\
\text { competências, como formuladores de políticas públicas, gerenciamento de } \\
\text { conflitos, profissionais de risco de TI, designer de mídia social e } \\
\text { especialistas em relacionamento com a comunidade. }\end{array}$ \\
\hline $\begin{array}{c}\text { Serviços, Infraestrutura e } \\
\text { Aplicações }\end{array}$ & $\begin{array}{l}\text { As cidades inteligentes devem manter soluções digitais, ferramentas, } \\
\text { aplicações, infraestrutura e níveis de serviços adequados para atendimento } \\
\text { às necessidades da comunidade, independente do fornecedor do serviço. }\end{array}$ \\
\hline
\end{tabular}

Fonte: [ISACA 2012] [ISACA 2018] [Reis et al. 2020]

Esses componentes de governança atuam como direcionadores para a estruturação e implementação de boas práticas de governança de TIC e são operacionalizados por artefatos [ISACA 2012] [SISP 2017] [ISACA 2018]. Com tais componentes em perspectiva, a metodologia para é apresentada a seguir.

\section{Metodologia de Pesquisa}

Quanto à natureza, essa pesquisa é aplicada, pois, abrange um estudo que contribui para a ampliação do conhecimento científico e prospecção de questões a serem investigadas sobre o tema [Gil 2010]. Quanto ao objetivo, é descritiva, pois busca uma maior familiaridade com o problema pesquisado, considerando que há poucas pesquisas acadêmicas [Gil 2010]. Quanto à abordagem, é qualitativa, pois busca analisar os dados intuitivamente. Quanto aos procedimentos, é bibliográfica e documental, pois baseou-se em informações disponíveis e em documentos solicitados [Gil 2010]. Nessa pesquisa os procedimentos metodológicos foram estruturados em cinco etapas: 1: Definição das informações necessárias, 2: Seleção dos Municípios, 3: Pesquisa das informações nos sites dos municípios, 4: Solicitação de informações aos municípios pela LAI e 5: Consolidação e análise das informações obtidas.

\section{1: Definição das informações necessárias}

Essa etapa teve como objetivo identificar as informações necessárias para compreender os requisitos de governança de TIC no contexto de cidades inteligentes tendo como referência o framework COBIT [ISACA 2012] [ISACA 2018b] [Reis et al. 2020], conforme Tabela 2.

\section{Tabela 2. Informações requeridas às prefeituras}

\begin{tabular}{|c|l|}
\hline Sol. & \multicolumn{1}{c|}{ Descrição } \\
\hline$\# 1$ & Plano Diretor de Tecnologia da Informação e Comunicação (PDTIC) instituído pelo município. \\
\hline$\# 2$ & Plano de investimentos em Tecnologia da Informação e Comunicação (TIC) instituído pelo município. \\
\hline$\# 3$ & Relação dos contratos de Tecnologia de Informação formalizados com terceiros. \\
\hline$\# 4$ & Plano de Capacitação de pessoal da área de Tecnologia da Informação e Comunicação (TIC) do município. \\
\hline$\# 5$ & Plano de Gestão de Riscos de Tecnologia de Informação e Comunicação (TIC) instituído pelo município. \\
\hline$\# 6$ & $\begin{array}{l}\text { Organograma da área de Tecnologia da Informação e Comunicações (TIC) do município que especifique a sua } \\
\text { estrutura organizacional, unidades vinculadas e subordinadas. }\end{array}$ \\
\hline$\# 7$ & $\begin{array}{l}\text { Estrutura dos processos de Tecnologia de Informação e Comunicação (TIC) do munícipio, identificando a } \\
\text { quantidade de pessoas envolvidas, atribuições, responsabilidades, tarefas e atividades desenvolvidas. }\end{array}$ \\
\hline$\# 8$ & $\begin{array}{l}\text { Relação dos Comitês de Tecnologia da Informação e Comunicação (TIC) vigentes no município, descrevendo } \\
\text { sua denominação, objetivo, competência e composição. }\end{array}$ \\
\hline$\# 9$ & $\begin{array}{l}\text { Documento do código de conduta do município que disciplina o uso/manuseio de recursos de tecnologia de } \\
\text { informação e comunicação pelos servidores da Prefeitura. }\end{array}$ \\
\hline$\# 10$ & $\begin{array}{l}\text { Relação dos Projetos Estratégicos do município que contemple o uso de uma solução tecnológica, relacionando } \\
\text { o nome do projeto, objetivo, valores envolvidos, benefícios previstos, tecnologias envolvidas, período de } \\
\text { conclusão e ações de monitoramento dos projetos. }\end{array}$ \\
\hline$\# 11$ & Plano ou instrumento similar adotado pela prefeitura para atendimento às diretrizes da Estratégia de Governança \\
\hline
\end{tabular}




\begin{tabular}{|c|c|}
\hline & Digital (EGD) instituída pelo Governo Federal. \\
\hline \#12 & $\begin{array}{l}\text { Relação dos fornecedores de serviços e soluções de Tecnologia da Informação e Comunicação para a prefeitura, } \\
\text { identificando os valores envolvidos, tipo de contratação e descrição dos serviços prestados. }\end{array}$ \\
\hline$\# 13$ & Plano ou Projeto adotado pelo município focado na privacidade de dados pessoais dos cidadãos. \\
\hline \#14 & $\begin{array}{l}\text { Relação dos canais e/ou recursos tecnológicos utilizados pela prefeitura para engajamento da comunidade nas } \\
\text { iniciativas digitais da cidade. }\end{array}$ \\
\hline \#15 & $\begin{array}{l}\text { Relação das ações estruturantes adotadas pela prefeitura para atender aos objetivos estratégicos do município } \\
\text { constantes do Plano Diretor. }\end{array}$ \\
\hline \#16 & $\begin{array}{l}\text { Relação das iniciativas estratégicas digitais na prefeitura para a transformação inteligente da cidade, com } \\
\text { identificação da área/setor responsável pela atividade. }\end{array}$ \\
\hline \#17 & $\begin{array}{l}\text { Relação das políticas de Tecnologia de Informação e Comunicação (TICs) adotadas na prefeitura, descrevendo } \\
\text { nome da política, diretrizes, objetivo e área responsável. }\end{array}$ \\
\hline \#18 & $\begin{array}{l}\text { Modelo de Governança de Tecnologia de Informação e Comunicação (TIC) adotado pela prefeitura, } \\
\text { identificando as atribuições e as responsabilidades da área de Governança de TIC, pessoas envolvidas e os } \\
\text { principais beneficios obtidos com o modelo. }\end{array}$ \\
\hline \#19 & $\begin{array}{l}\text { Relação das Parcerias Públicas Privadas (PPP), Acordos Locais ou Internacionais, seja com Governos ou } \\
\text { Empresas especializadas firmados pela prefeitura que envolvem atividades relacionadas ao uso de recursos de } \\
\text { Tecnologia de Informação e Comunicação (TIC). }\end{array}$ \\
\hline \#20 & $\begin{array}{l}\text { Relação dos indicadores instituídos pela prefeitura para mensurar o desempenho da área de Tecnologia de } \\
\text { Informação e Comunicação (TIC). }\end{array}$ \\
\hline
\end{tabular}

Fonte: [ISACA 2012] [ISACA 2018b] [Reis et al. 2020]

Essa documentação foi utilizada para efetuar o levantamento de requisitos de boas práticas de governança de TIC no contexto de cidades inteligentes.

\section{2: Seleção dos Municípios}

O Brasil tem 5.570 municípios, dentre estes, 92 estão localizados no Estado do Rio de Janeiro [IBGE 2017]. Esse estudo limitou-se a uma amostra de dez municípios localizados no estado do RJ. A seleção dos municípios baseou-se no ranking das cidades pelo índice IGM-CFA (Índice de Governança Municipal Conselho Federal de Administração), que consiste em uma métrica anual de governança pública para os municípios brasileiros baseada em três dimensões: Finanças, Gestão e Desempenho [CFA 2018]. O IGM-CFA foi estruturado a partir de dados secundários que considera as áreas saúde, educação, planejamento urbano, articulação institucional, gestão fiscal, habitação, recursos humanos e violência. O IGM-CFA segrega os municípios em três critérios (clusters): tamanho da população, renda per capita e área territorial do município. Esses clusters facilitam o agrupamento entre os municípios porque contemplam características similares em que cada subgrupo de municípios apresenta um "porte" (número de habitantes) e um "PIB per capita" similares.

Nesse trabalho, para definição da amostra, foi utilizado o cluster 8 (oito) que considera população acima de 100.000 habitantes e PIB per capita acima de $\mathrm{R} \$ 28.900$. O ranking da consulta ao IGM-CFA [CFA, 2018] em 19/12/2019 obteve o seguinte resultado do $1^{\circ}$ ao $10^{\circ}$, respectivamente: Niterói, Rio de Janeiro, Petrópolis, Resende, Angra dos Reis, Volta Redonda, Macaé, Rio das Ostras, Campo dos Goytacazes e Queimados.

\section{3: Pesquisa das informações nos sites dos municípios}

Essa etapa compreendeu analisar se as informações requeridas, apresentadas na Tabela 2, estavam disponíveis nos sites oficiais dos municípios. 


\section{4: Solicitação de informações aos municípios pela LAI}

Essa etapa consistiu em solicitar as informações constantes da Tabela 2 aos municípios com base na Lei de Acesso a Informações [Brasil 2011], no portal da transparência de cada prefeitura.

\section{5: Consolidação e análise das informações obtidas}

Essa etapa consistiu em consolidar e analisar as informações nos sites dos municípios (Etapa 3) e nas respostas obtidas às requisições da LAI (Etapa 4).

\section{Discussão dos Resultados}

\subsection{Análise das informações disponibilizadas pelos municípios}

Não foi possível identificar nos sites oficiais das prefeituras as informações constantes da Tabela 2. Assim, optou-se por solicitá-las no portal de transparência de cada município. Esse procedimento teve como objetivo não incorrer em falhas na localização, identificação ou interpretação dos dados. A Figura 1 sintetiza o resultado dos vinte critérios de informações avaliados nos dez municípios.

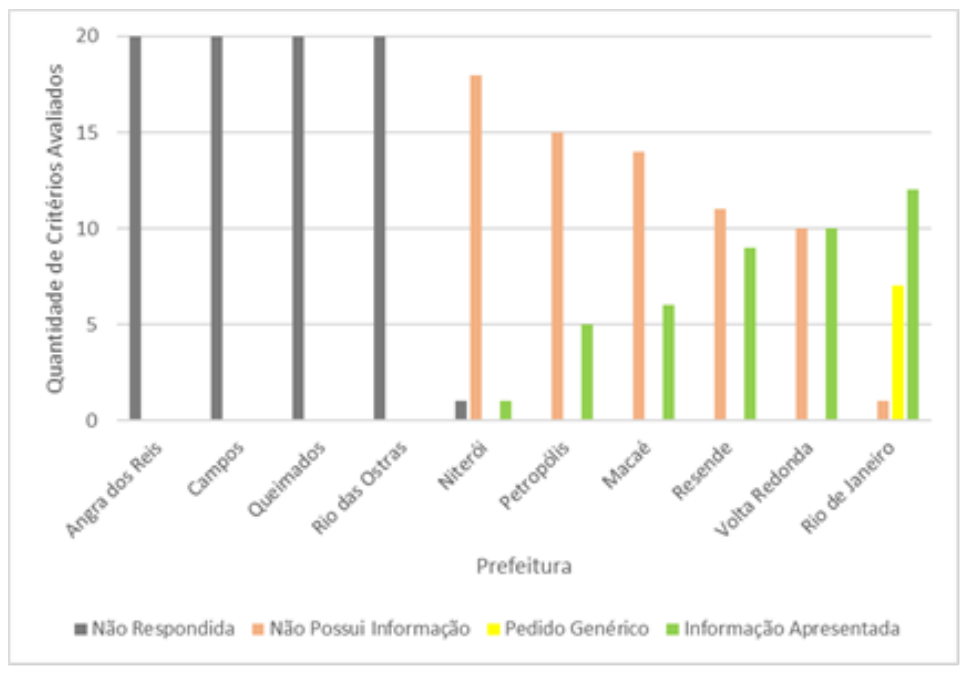

Figura 1. Visão geral das informações solicitadas por município

Fonte: Coleta de dados pelos autores

Em quatro municípios não se obteve resposta às solicitações, os quais foram Angra dos Reis, Campos dos Goytacazes, Queimados e Rio das Ostras. As requisições às prefeituras de Angra dos Reis e Campos dos Goytacazes encontram "Em andamento" desde a data da solicitação em janeiro, portanto, sem atendimento no prazo legal. Não houve manifestação e atendimento da prefeitura de Queimados para as solicitações efetuadas em fevereiro, também não atendendo ao prazo legal. O portal da transparência da prefeitura de Rio das Ostras não respondeu às requisições de consulta e apresentou a mensagem "This site can't be reached". Efetuamos contato pelo canal Fale Conosco no telefone (22)2771-1515 e na URL https://www.riodasostras.rj.gov.br/fale-conosco/, porém sem sucesso. 
Para as seis prefeituras restantes, Niterói informou que em dezoito itens não possuía as informações solicitadas, um item de informação foi apresentado e para outro não obtivemos resposta. Foi informado que alguns documentos estão em elaboração, mas sem previsão de finalização. A prefeitura de Petrópolis apresentou cinco itens de informação e para os outros quinze informou que não possuía os documentos. A prefeitura de Macaé apresentou seis itens de informação e não possuía as documentações para os quatorze restantes. A prefeitura de Resende apresentou onze itens solicitados e para nove informou que não possuía os documentos. A prefeitura de Volta Redonda respondeu a todas as solicitações, porém algumas informações apresentadas não estavam relacionadas aos pedidos. Apesar da interposição de recurso no portal de transparência, a prefeitura negou atendimento. Dez itens de informação foram aceitos e os outros restantes não foram. A prefeitura do Rio de Janeiro apresentou doze itens de informação, para um item foi informado que não possuía a documentação e para sete itens classificou o pedido como genérico, sem aceitar o recurso interposto.

O resultado de cada critério de informação avaliado por município, a exceção dos quatro que não responderam, está consignado na Tabela 3 . Nessa tabela, as células marcadas com " $\checkmark$ " e na cor verde indicam que os documentos foram apresentados/aceitos; as células marcadas com " $x$ " e na cor rosa indicam que os documentos não foram apresentados/aceitos; as células marcadas com ":)" e na cor amarelo indicam que as solicitações de informação foram consideradas genéricas e não atendidas pela prefeitura; e a célula marcada com "•" na cor cinza indica que a prefeitura não respondeu o item solicitado.

Tabela 3. Avaliação das informações por município

\begin{tabular}{|c|c|c|c|c|c|c|}
\hline Artefato/Prefeitura & Niterói & Macaé & Petrópolis & Resende & $\begin{array}{c}\text { Rio de } \\
\text { Janeiro }\end{array}$ & $\begin{array}{c}\text { Volta } \\
\text { Redonda }\end{array}$ \\
\hline Ações Estruturantes & $x$ & $\checkmark$ & $\checkmark$ & $\checkmark$ & $\checkmark$ & $\checkmark$ \\
\hline Relação de Contratos de TIC & $x$ & $\checkmark$ & $\checkmark$ & $\checkmark$ & (2) & $\checkmark$ \\
\hline Relação de Fornecedores de TIC & $x$ & $\checkmark$ & $\checkmark$ & $\checkmark$ & (2) & $\checkmark$ \\
\hline Canais para Engajamento & $x$ & $\checkmark$ & $\checkmark$ & $x$ & $\checkmark$ & $\checkmark$ \\
\hline Plano de Capacitação de TIC & $x$ & $x$ & $x$ & $\checkmark$ & $\checkmark$ & $\checkmark$ \\
\hline Indicadores de TIC & $x$ & $x$ & $x$ & $\checkmark$ & $\checkmark$ & $\checkmark$ \\
\hline Plano de Investimentos de TIC & $x$ & $\checkmark$ & $x$ & $x$ & $\checkmark$ & $\checkmark$ \\
\hline Iniciativas estratégicas & + & $\checkmark$ & $x$ & $\checkmark$ & (:) & $\checkmark$ \\
\hline Relação de Projetos Estratégicos & $x$ & $x$ & $x$ & $\checkmark$ & (:) & $\checkmark$ \\
\hline Organograma de TIC & $x$ & $x$ & $x$ & $\checkmark$ & (:) & $\checkmark$ \\
\hline Comitês de TIC & $\checkmark$ & $x$ & $x$ & $x$ & $\checkmark$ & $x$ \\
\hline Código de Conduta & $x$ & $x$ & $\checkmark$ & $x$ & $\checkmark$ & $x$ \\
\hline Estratégia de Governança Digital & $x$ & $x$ & $x$ & $\checkmark$ & $\checkmark$ & $x$ \\
\hline Plano Diretor de TIC & $x$ & $x$ & $x$ & $x$ & $\checkmark$ & $x$ \\
\hline Plano de Gestão de Riscos de TIC & $x$ & $x$ & $x$ & $x$ & $\checkmark$ & $x$ \\
\hline Políticas de TIC & $x$ & $x$ & $x$ & $x$ & $\checkmark$ & $x$ \\
\hline Modelo de Governança de TIC & $x$ & $x$ & $x$ & $x$ & $\checkmark$ & $x$ \\
\hline Parcerias e Acordos de TICs & $x$ & $x$ & $x$ & $x$ & (2) & $x$ \\
\hline Processos de TIC & $x$ & $x$ & $x$ & $x$ & (2) & $\bar{x}$ \\
\hline Plano de Privacidade & $x$ & $x$ & $x$ & $x$ & $x$ & $x$ \\
\hline
\end{tabular}

Fonte: Coleta de dados pelos autores

Essa tabela revela que algumas prefeituras dispõem de evidências que adotam boas práticas de governança de TIC e que determinadas práticas estão presentes em mais de uma prefeitura. Todavia, há práticas que não são adotadas em nenhuma prefeitura. A seguir, os dados são apresentados à luz dos componentes do sistema de governança [ISACA 2018] [Reis et al. 2020]. 


\subsection{Análise das informações à luz dos componentes do sistema de governança}

O resultado da análise à luz dos componentes de governança consta da Tabela 4 .

Tabela 4. Análise dos resultados à luz dos componentes de governança

\begin{tabular}{|c|c|}
\hline Componente & Resultados das Análises \\
\hline $\begin{array}{l}\text { Princípios, } \\
\text { Políticas e } \\
\text { Modelos }\end{array}$ & $\begin{array}{l}\text { Cinco prefeituras apresentaram a relação de ações estruturantes para } \\
\text { atender aos objetivos do Plano Diretor. Essa prática indica que essas } \\
\text { prefeituras utilizam o Plano Diretor como instrumento para as estratégias } \\
\text { municipais, situação que auxilia o planejamento estratégico institucional } \\
\text { das cidades. As diretrizes de governança digital do governo federal [Brasil } \\
\text { 2018] são utilizadas como insumo para promover o desenvolvimento local } \\
\text { nas prefeituras de Resende e do Rio de Janeiro utilizam, situação que } \\
\text { revela um baixo alinhamento entre as instâncias federais e municipais para } \\
\text { a governança. No contexto de cidades inteligentes proposto por [Gil- } \\
\text { Gárcia et al. 2015], na perspectiva de tecnologia, essa integração auxilia a } \\
\text { estratégia das cidades para alcançar seus objetivos por mecanismos de } \\
\text { governança. A prefeitura do Rio de Janeiro foi a única que apresentou os } \\
\text { artefatos Plano Diretor de TIC (PDTIC), Políticas de TIC, Plano de Gestão } \\
\text { de Riscos de TIC e Modelo de Governança, o que revela baixa } \\
\text { representatividade do uso desses artefatos nas demais prefeituras. Segundo } \\
\text { [ISACA 2018b], esses artefatos auxiliam no fortalecimento da estrutura de } \\
\text { governança de TIC das instituições. Na perspectiva de cidades inteligentes, } \\
\text { esses artefatos auxiliam na concepção de diretrizes, planejamento } \\
\text { estratégico, politicas, regras, princípios e procedimentos relacionados às } \\
\text { TICs para atingimento das metas das cidades. }\end{array}$ \\
\hline Processos & $\begin{array}{l}\text { Quatro prefeituras evidenciaram boas práticas na gestão de bens e serviços } \\
\text { de TIC relacionadas à contratação de soluções e fornecedores. Nenhuma } \\
\text { prefeitura possui uma estrutura definida para os processos de TIC com } \\
\text { identificação da quantidade de pessoas envolvidas, atribuições, } \\
\text { responsabilidades, tarefas e atividades desenvolvidas, o que dificulta uma } \\
\text { gestão efetiva do ambiente e representa um desafio para a estruturação da } \\
\text { governança baseada em processos, práticas de trabalho e ambiente cultural } \\
\text { [Weill and Broadbent 2000]. Na perspectiva de cidades inteligentes, as } \\
\text { prefeituras devem estruturar um modelo de referência de processos de TIC } \\
\text { alinhado aos seus objetivos estratégicos, com propósitos, padrões e metas. }\end{array}$ \\
\hline $\begin{array}{c}\text { Serviços, } \\
\text { Infraestrutura e } \\
\text { Aplicações }\end{array}$ & $\begin{array}{l}\text { quatro prefeituras utilizam canais para engajamento da comunidade nas } \\
\text { iniciativas digitais da cidade, situação relevante sob a perspectiva de } \\
\text { governança participativa no contexto de cidades inteligentes. A prefeitura } \\
\text { de Resende possui uma URL específica para o projeto Resende Inteligente, } \\
\text { destinado à transformação inteligente do município. Na perspectiva de } \\
\text { cidades inteligentes, as prefeituras devem manter soluções digitais, } \\
\text { ferramentas, aplicações, infraestrutura e níveis de serviços para } \\
\text { atendimento às necessidades locais. }\end{array}$ \\
\hline $\begin{array}{l}\text { Pessoas, } \\
\text { Habilidades e } \\
\text { Competências } \\
\text { Cultura, Ética e } \\
\text { Comportamentos }\end{array}$ & $\begin{array}{l}\text { três prefeituras apresentaram um plano de capacitação de TIC para os } \\
\text { colaboradores e duas prefeituras possuem código de conduta para nortear } \\
\text { os conhecimentos dos profissionais que atuam na área de TIC. Na } \\
\text { perspectiva de cidades inteligentes, esses componentes abrangem a } \\
\text { cultura, a ética e o comportamento dos cidadãos e dos profissionais de } \\
\text { TIC, requisitos a serem considerados no planejamento e no } \\
\text { desenvolvimento de serviços de TIC. }\end{array}$ \\
\hline $\begin{array}{c}\text { Estrutura } \\
\text { Organizacional }\end{array}$ & $\begin{array}{l}\text { duas prefeituras apresentaram o organograma de TIC e duas possuem um } \\
\text { Comitê de TIC institucionalizado. Por exemplo, a prefeitura de Niterói } \\
\text { possui um Comitê Estratégico de Tecnologia da Informação (CETI), } \\
\text { composto por membros consultivos (técnicos) e deliberativos (gestores), } \\
\text { que tem o objetivo de controlar as contratações de TI, com ênfase em } \\
\text { aspectos técnicos e em custo x benefício. Na perspectiva de cidades } \\
\text { inteligentes, a estrutura administrativa deve contemplar níveis apropriados }\end{array}$ \\
\hline
\end{tabular}




\begin{tabular}{|l|l|}
\hline & $\begin{array}{l}\text { de responsabilidade, autoridade e atribuições para as decisões sobre } \\
\text { soluções e investimentos de TIC. }\end{array}$ \\
\hline \multirow{7}{*}{ Informações } & $\begin{array}{l}\text { Nenhuma prefeitura apresentou o Plano de Privacidade de Dados, o que } \\
\text { demonstra falta de iniciativa para atender aos requisitos da LGPD - Lei } \\
\text { Geral de Privacidade de Dados Pessoais [Brasil 2018b]. Nenhuma } \\
\text { prefeitura apresentou evidência de Parcerias e/ou Acordos de TICs, o que } \\
\text { revela que não há iniciativas para apoiar os serviços de Parceria Público- } \\
\text { Privada (PPP). Na perspectiva de cidades inteligentes, os tomadores de } \\
\text { decisão necessitam de dados, informações e conhecimento compartilhado } \\
\text { entre as partes interessadas, de forma transparente e segura, com requisitos } \\
\text { de confidencialidade e privacidade. }\end{array}$ \\
\hline
\end{tabular}

Fonte: Coleta de dados pelos autores

\section{Conclusões e Trabalhos Futuros}

Considerando tanto as definições de cidades inteligentes quanto os conceitos de governança de TIC, para que as prefeituras se tornem efetivamente inteligentes, há uma série de desafios que precisam ser superados, a começar pela adoção de boas práticas de governança de TIC. Isso é devido em função das TICs representarem uma dimensão do framework de cidade inteligente [Gil-Gárcia et al. 2015]. Assim, as prefeituras necessitam estruturar e implementar boas práticas de governança para aprimorar os processos internos e entregar valor aos cidadãos. Uma motivação para o desenvolvimento da metodologia na forma de análise exploratória baseou-se no levantamento efetuado por [Reis et al. 2020] que evidenciou que há uma quantidade limitada de publicações em Sistemas de Informação que abordaram boas práticas de governança de TIC com uso do framework COBIT no contexto de cidades inteligentes.

Essa pesquisa apresentou uma análise exploratória da adoção de instrumentos de boas práticas para a governança de TIC em dez prefeituras do estado do Rio de Janeiro sob o olhar do conceito de cidades inteligentes. Pudemos observar nesse estudo exploratório que (i) quatro dos dez municípios não apresentaram informações sobre adoção de boas práticas de governança de TIC nem em seus sites e nem responderam aos questionamentos realizados em seus portais de transparência; e (ii) nos seis municípios em que as informações foram encontradas, muitas boas práticas não são realizadas. Deve-se observar que os dez municípios analisados, dentre os 92 no estado do RJ, apresentam os maiores índices de governança municipal no estado. Entende-se que essa pesquisa contribui para o enriquecimento científico sobre o tema e pode auxiliar os governos na administração e no gerenciamento dos municípios no contexto de cidades inteligentes usando boas práticas de governança de TIC, uma vez que os resultados evidenciaram a necessidade de adoção de requisitos de governança.

Uma limitação desse trabalho refere-se ao fato de que os documentos apresentados foram analisados, à luz de sua existência, sob aspectos de conformidade em relação aos requisitos estabelecidos em guias de boas práticas de governança de TIC, não sendo objeto desse estudo a sua avaliação e aderência ao contexto e à realidade de cada prefeitura, situação que pode ser objeto de estudos futuros. Estudos futuros também podem ampliar a quantidade da amostra, incluindo uma análise de municípios em outros estados com altos índices de governança municipal e com projetos de cidades inteligentes. Além disso, uma 
análise mais aprofundada incluindo surveys com especialistas e entrevistas com membros dos governos municipais também podem ser realizadas para melhor entendimento do uso de boas práticas de governança de TIC nos municípios. Ainda, artefatos não contemplados nesse estudo podem ser avaliados, tais como os presentes no COBIT 2019 [ISACA 2018b].

\section{Referencias}

Ahvenniemi, H., Huovila, A., Pinto-Seppa, I., Airaksinen, M. (2017). What are the differences between sustainable and smart cities? Cities, 60, 234-245.

Bianchi, I. S., Sousa, R. D., Pereira, R., Luciano, E. (2017). IT Governance Structures in Brazilian, Dutch and Portuguese Universities. Procedia Computer Science, 121, 927-933.

Bolívar, M. P. R. (2015). Characterizing the Role of Governments in Smart Cities: A Literature Review. Smarter as the New Urban Agenda, 49-71.

Boscarioli, C.; Araujo, R. M.; Maciel, R. S. P. (2017). I GranDSI BR Grand Research Challenges in Information Systems in Brazil 2016-2026. Special Committee on Information Systems (CE SI). Brazilian Computer Society.

Brasil, IBGE (2017). Instituto Brasileiro de Geografia e Estatística, IBGE. Disponível em https://cidades.ibge.gov.br/brasil/panorama.

Brasil, Lei $\mathrm{n}^{\circ} 10.257$ (2001). Estatuto da Cidade. Disponível em http://www.planalto.gov.br/ccivil_03/leis/leis_2001/110257.htm.

Brasil, Lei $\mathrm{n}^{\circ} 12.527$ (2011). Acesso a informações. Disponível em http://www.planalto.gov.br/ccivil_03/_ato2011-2014/2011/lei/112527.htm.

Brasil, Ministério do Planejamento, Desenvolvimento e Gestão. (2018). Estratégia de Governança Digital.

Brasil, Lei n 13.709, (2018b). Lei Geral de Proteção de Dados. Disponível em www.planalto.gov.br.

CFA, Índice de Governança Municipal. IGM-CFA. (2018). Ranking dos Grupos IGM-CFA. Disponível em http://www.igm.cfa.org.br/.

Caragliu, A., Del Bo, C., Nijkamp, P. (2011). Smart cities in Europe. Journal of Urban Technology, 18(2), 65-82.

Connolly, N., Maccani, G., Donnellan, B. (2017). IT Governance in Smart Cities: A Conceptual Framework. ICIS.

De Haes, S., Van Grembergen, W. (2009). An Exploratory Study into IT Governance Implementations and its Impact on Business/IT Alignment. IS Management. 26. 123-137. 10.1080/10580530902794786.

De Haes, S., Van Grembergen, W., Debreceny, R. (2013). COBIT 5 and Enterprise Governance of Information Technology: Building Blocks and Research Opportunities. Journal of Information Systems.

De Haes S., Van Grembergen W., Joshi A., Huygh T. (2019) COBIT as a Framework for Enterprise Governance of IT. In: Enterprise Governance of Information Technology. Management for Professionals. Springer, Cham. 
GIL, A. C. (2010). Como elaborar projetos de pesquisa. 5. ed. São Paulo: Atlas.

Gil-Gárcia, J. R.; Pardo, T.; Nam, T. (2015) What makes a city smart? Identifying core components and proposing an integrative and comprehensive conceptualization. Information Polity, vol. 20, no. 1, pp. 61-87.

Héroux, S.; Fortin, A. (2017). Exploring the influence of executive management diversity on IT governance. CHAIRE DínformationFinanciére et Organisationnelle ESQ UQÀM, (514), 1-33.

Hollands, R. G. (2008). Will the real smart city please stand up? Intelligent, progressive or entrepreneurial? City, 12(3), 303-320.

Huang, R., Zmud, R.W., Price, R.L. (2010). Influencing the effectiveness of IT governance practices through steering committees and communication policies, European Journal of Information Systems, 19(3), pp.288-302.

ISACA. (2012). COBIT 5: A Business Framework for the Governance and Management of Enterprise IT. Rolling Meadows, IL: ISACA.

ISACA. (2018). COBIT 2019 Framework: Introduction and Methodology. Rolling Meadows, IL: ISACA.

ISACA. (2018b). COBIT 2019 Framework: Governance and Management Objectives. Rolling Meadows, IL: ISACA.

Joshi, A., Huygh, T., Haes, S., Grembergen, W. (2018). An Empirical Assessment of Shared Understanding in IT Governance Implementation. HICSS, 2018.

Jucevicius, R., Patašiene, I., Patašius, M. (2014). Digital Dimension of Smart City: Critical Analysis. Procedia - Social and Behavioral Sciences, 156.

Kobayashi, A. R. K., Kniess, C. T., Serra, F. A. R., Ferraz, R. R. N., Ruiz, M. S. (2017). Cidades inteligentes e sustentáveis: estudo bibliométrico e de informações patentárias. International Journal of Innovation, 5(1), 77-96.

Nam, T., Pardo, T.A. (2011). Conceptualizing smart city with dimensions of technology, people, and institutions. In: 12th Annual International Digital Government Research Conference. ACM.

Pereira, G. V., Parycek, P., Falco, E., Kleinhans, R. (2018). Smart governance in the context of smart cities: A literature review. Information Polity.

Reis, L. Diogo, Ferreira, S. B. L., Bernardini, F. C., Cappelli, C. (2020). Towards a COBIT5 Approach to ICT Governance Requirements in Smart Cities. ICEGOV. $13^{\text {th }}$ International Conference on Electronic Governance.

SISP, Guia de Governança de TIC. (2017). Ministério do Planejamento, Desenvolvimento e Gestão. Disponível em http://www.sisp.gov.br/.

Weill, P., Broadbent, M. (2000). Managing IT infrastructure: a strategic choice. In: Zmud, R., (Ed.), Framing the Domains of IT Management: Projecting the Future Through the Past, Pinnaflex Educational Resources, Cincinnati, OH.

Weill, P. and Ross, J. W. (2004). IT Governance on One Page. MIT Sloan Working Paper No. 4517-04; CIS Research Working Paper No. 349. Available at SSRN: https://ssrn.com/abstract $=664612$. 